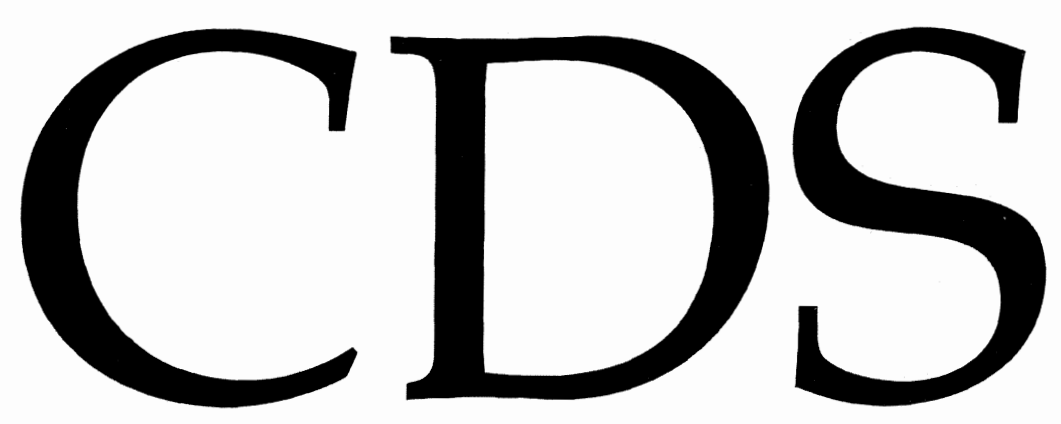

TECHNICAL MEMORANDUM NO. CIT-CDS 92-002

October 14, 1992

"Nilpotent Bases for a Class of Non-Integrable Distributions with Applications to Trajectory Generation for Nonholonomic Systems"

Richard M. Murray

Control and Dynamical Systems California Institute of Technology Pasadena, CA 91125 


\title{
NILPOTENT BASES FOR A CLASS OF NON-INTEGRABLE DISTRIBUTIONS WITH APPLICATIONS TO TRAJECTORY GENERATION FOR NONHOLONOMIC SYSTEMS
}

\author{
RICHARD M. MURRAY \\ Division of Engineering and Applied Science \\ California Institute of Technology \\ Pasadena, California 91125 \\ murray@design.caltech.edu
}

7 October 1992

\begin{abstract}
This paper develops a constructive method for finding a nilpotent basis for a special class of smooth nonholonomic distributions. The main tool is the use of the Goursat normal form theorem which arises in the study of exterior differential systems. The results are applied to the problem of finding a set of nilpotent input vector fields for a nonholonomic control system, which can then used to construct explicit trajectories to drive the system between any two points. A kinematic model of a rolling penny is used to illustrate this approach. The methods presented here extend previous work using "chained form" and cast that work into a coordinate-free setting.
\end{abstract}

\section{INTRODUCTION}

This work is motivated by the recent interest in trajectory generation for mechanical systems with nonholonomic constraints. Consider the problem of steering a mechanical system with $n$-dimensional configuration space $M$ from an initial configuration $x^{0}$ to a final configuration $x^{1}$, subject to a set of independent kinematic constraints having the form

$$
<\omega_{j}(x), \dot{x}>=0 \quad j=1, \cdots, k .
$$

The $\omega_{j}$ 's are a basis for a codistribution on $M$ which restricts the velocity of the system to be zero in certain directions. We assume the $\omega_{j}$ 's are smooth and linearly independent over the ring of smooth functions. Such constraints can arise when two surfaces roll against each other, such as the rolling between a wheel and the road, or in space-based systems where the total angular momentum of the system

Research supported in part by a grant from the Powell Foundation.. 
is conserved (although strictly speaking this latter case is not a "constraint", it can be treated with the same set of tools).

To study such a system, we convert the path planning problem into a control problem. Let $\Delta$ be a distribution of dimension $m=n-k$ which is annihilated by the constraints. We represent this distribution with respect to a local basis of vector fields:

$$
\Delta=\operatorname{span}\left\{g_{1}, g_{2}, \cdots, g_{m}\right\} \quad g_{i}(x) \in T_{x} M .
$$

In coordinates, the constraint one-forms can be written as an $k \times n$ matrix and the $g_{i}$ 's are a basis for the right null space of this matrix. The path planning problem can then be restated as finding an input function, $u(t) \in \mathbb{R}^{m}$ such that the control system

$$
\Sigma: \quad \dot{x}=g_{1}(x) u_{1}+\cdots+g_{m}(x) u_{m}
$$

is driven from $x^{0}$ to $x^{1}$. As a consequence of our assumptions on the $\omega_{j}$ 's, the $g_{i}$ 's are also smooth and linearly independent. We restrict the analysis to a local coordinate chart on $M$.

The conditions for the existence of a path between two configurations is given by Chow's theorem. We let $[f, g]$ be the Lie bracket between two vector fields,

$$
[f, g]=\frac{\partial g}{\partial x} f-\frac{\partial f}{\partial x} g
$$

and define the involutive closure of a distribution $\Delta$ as the closure of $\Delta$ under Lie bracketing. Chow's theorem asserts that if the involutive closure of the distribution associated with equation (3) spans $T_{x} M$ at each configuration, the system can be steered between any two configurations. It is not apparent how the path can be explicitly constructed: the trajectory generation problem is to explicitly construct paths between two points which everywhere satisfy the constraints.

A set of kinematic constraints is holonomic if the constraints restrict the motion of the system to a submanifold of dimension $n-k$. In this case, the constraints on the system can be rewritten as an algebraic constraint on the configuration variables $x$. The constraints are nonholonomic if they do not constrain the system to lie on a submanifold of the same dimension as the input space. In particular, we are most interested in constraints which are maximally nonholonomic: any point in the configuration space can be reached. This is equivalent to saying that the corresponding control system is controllable. If the constraints are not maximally nonholonomic, the system can still be analyzed by restricting the initial and final configurations to lie on the same leaf of the foliation generated by the distribution. A control system which has the form given in equation (3) is called a nonholonomic control system.

The general problem of finding a feasible trajectory for a nonholonomic system is difficult. For certain application areas-such as mobile robotics-fast, efficient algorithms are required and one is often willing to sacrifice conditions such as optimality if the tradeoff is finding a reasonable path in an efficient fashion (see [11] 
for a review of nonholonomic motion planning in this context). Motivated in part by these applications, there have been recent efforts to understand and exploit the structure of nonholonomic systems using tools from differential geometric control theory. In particular, the use of nilpotent vector fields has appeared repeatedly in the work of Lafferriere and Sussmann [9], Jacob [7, 8], and the author [12].

We recall the definition of a nilpotent basis for a distribution. Given a basis of vector fields $\left\{g_{1}, \cdots, g_{m}\right\}$, we define the length of a Lie product recursively as

$$
\begin{aligned}
l\left(g_{i}\right) & =1 \\
l([A, B]) & =l(A)+l(B)
\end{aligned}
$$

where $A$ and $B$ are themselves Lie products. Alternatively, $l(A)$ is the number of generators in the expansion for $A$. A basis is nilpotent if there exists an integer $k$ such that all Lie products of length greater than $k$ are zero. $k$ is called the order of nilpotency. The advantage of using a nilpotent basis to represent a distribution is that certain computations are greatly simplified since high order brackets are identically zero.

Recently a very elegant and general motion planning method for nilpotent systems has been developed by Lafferriere and Sussmann [9]. The main tool in their method is the Baker-Campbell-Hausdorff formula, which describes the composition of flows of vector fields as a single flow:

$$
\phi^{X_{1}} \circ \phi^{X_{2}}=\phi^{X_{1}+X_{2}+\frac{1}{2}\left[X_{1}, X_{2}\right]+\cdots}
$$

Their method consists of first converting a desired motion into a flow of the form:

$$
\phi^{\alpha_{1} X_{1}+\alpha_{2} X_{2}+\alpha_{3}\left[X_{1}, X_{2}\right]+\cdots}
$$

and then using the Baker-Campbell-Hausdorff formula, in combination with a $\mathrm{P}$. Hall basis for the Lie algebra generated by $\left\{X_{i}\right\}$, to choose piecewise constant inputs that achieve this flow. If the system is nilpotent, the Baker-Campbell-Hausdorff formula has a finite number of terms and it can be shown that the process terminates. A similar approach has been used by Jacob [7, 8] using a different choice of basis for the Lie algebra, namely a Lyndon basis.

Somewhat related to these approaches, our own work has focused on the use of specific choices of coordinates such that the control system can be explicitly integrated by quadratures. The basic technique is to search for a feedback transformation which puts the system into a special nilpotent form (called chained form), and then use sinusoids at integrally related frequencies to drive the system between states [13]. In the case of two-input systems, constructive sufficient conditions for the existence of a nilpotent basis for the associated distribution were given in in [12]. In this paper we extend that work to give a simple rank condition which guarantees the existence of a nilpotent basis for two-dimensional distribution and show how the resulting basis can be applied to solve the trajectory generation problem.

The general problem of finding a nilpotent basis for a distribution has been studied by Hermes et al. [6]. They present a set of necessary conditions for the existence of a local nilpotent basis and give two sets of sufficient conditions, one based on 
solving a partial differential equation and the other based on Darboux's theorem. The result presented here also relies on solving a partial differential equation, but the existence of a solution is guaranteed by the rank condition.

This paper is organized as follows: Section 2 presents a rank test for determining if a nonholonomic distribution admits a nilpotent basis. We concentrate on the twoinput case and present a constructive procedure for finding such a basis. Section 3 gives an application of these results to the trajectory generation problem described above. We use as an example the case of a rolling penny [1]. Finally, we discuss the implications of these results to other problems and describe areas of future work.

\section{NilPOTENT BASES FOR A CLASS OF NONHOLONOMIC SYSTEMS}

In this section we present a set of necessary and sufficient conditions for a twoinput nonholonomic control system to have a nilpotent basis of a certain special form. The conditions lead to a constructive method of finding a feedback transformation which converts the nonholonomic system into a nilpotent, nonholonomic system. This extends the work initiated in [13] by giving a set of necessary and sufficient conditions for the existence of a feedback transformation which converts a system into chained form.

2.1. Pfaffian Systems. The proofs in this paper make extensive use of tools from the theory of exterior differential systems. We present a brief overview of the relevant concepts here. A detailed description can be found in the monograph by Bryant et al. [2].

Let $M$ be a manifold of dimension $n$ with cotangent bundle $T^{*} M$; let $\Omega^{p}(M)$ denote the set of smooth exterior $p$-forms on $M$. Define $\Omega^{*}(M)$ as the set of smooth exterior differential forms of all orders on a manifold $M, \Omega^{*}(M)=\bigoplus \Omega^{p}(M)$. An exterior differential system is given by an ideal $\mathcal{I} \subset \Omega^{*}(M)$ that is closed under exterior differentiation.

A Pfaffian system is an exterior differential system which is generated by a set of linearly independent one-forms

$$
I=\left\{\alpha^{1}, \ldots, \alpha^{s}\right\} .
$$

The associated ideal has the form

$$
\mathcal{I}=\left\{\omega \wedge \theta: \omega \in I, \theta \in \Omega^{*}(M)\right\} .
$$

We call $s$ the dimension of the system (assumed constant).

The exterior derivative induces a mapping $\delta: I \rightarrow \Omega^{2}(M) / I$ :

$$
\delta: \lambda \mapsto d \lambda \bmod I \in \Omega^{2}(M) .
$$

The mapping $\delta$ is a linear mapping over $C^{\infty}(M)$ :

$$
\begin{aligned}
\delta(f \alpha+g \beta) & =d f \wedge \alpha+f d \alpha+d g \wedge \beta+f d \beta \bmod I \\
& =f d \alpha+g d \beta \bmod I \\
& =f \delta(\alpha)+g \delta(\beta) .
\end{aligned}
$$


It follows that the kernel of $\delta$ is a subbundle of $\Omega(M)$ (i.e., at each point $p \in M$, the kernel of $\delta$ is a linear subspace of $\left.T_{p}^{*} M\right)$. We call this subbundle $I^{(1)}$, the first derived system of $I$ :

$$
I^{(1)}=\operatorname{ker} \delta=\{\lambda \in I: d \lambda \bmod I \equiv 0\} .
$$

We represent $I^{(1)}$ using a basis of one-forms and hence $I^{(1)}$ is a Pfaffian system.

Since $I^{(1)}$ is itself a Pfaffian system, we can continue this construction and generate a filtration

$$
I=I^{(0)} \supset I^{(1)} \supset \cdots \supset I^{(N)} .
$$

If the dimension of each $I^{(i)}$ is constant, then this construction terminates for some finite integer $N$. In this case, we call equation (4) the derived flag of $I$ and $N$ the derived length.

The derived flag describes the integrability properties of the ideal generated by $I$. If $I$ is completely integrable, then by Frobenius's theorem we have $I^{(1)}=I^{(0)}$, i.e. the derived length of the flag is zero. In fact, $I^{(N)}$ is always integrable since by definition $d I^{(N)} \bmod I^{(N)} \equiv 0 . I^{(N)}$ is the largest integrable subsystem contained in $I$. Thus if $I^{(N)}$ is not empty, then there exist functions $h_{1}, \ldots, h_{r}$ such that $\left\{d h_{i}\right\} \subset\{I\}$. In the context of control theory, this means that the system is not controllable since there exist algebraic functions which provide a foliation of the state space and it is impossible to move from one leaf of the foliation to another. The converse of this controllability result is provided by Chow's Theorem:

Theorem 1 (Chow). Let $I=\left\{\alpha^{1}, \ldots, \alpha^{s}\right\}$ represent $a$ set of constraints and assumed that the derived flag of the system exists. Then, there exists a path $x(t)$ between any two points satisfying $\alpha^{i}(x) \dot{x}=0$ for all $i$ if and only if there exists an $N$ such that $I^{(N)}=0$.

2.2. Goursat normal form. The main results of this section are a direct consequence of the following theorem, whose proof can be found in Bryant et al. [2, pp. 54-57].

Theorem 2 (Goursat normal form). Let $U$ be an open subset of $\mathbb{R}^{n}$ and $I=$ $\left\{\alpha^{1}, \ldots, \alpha^{s}\right\}$ be a collection of smooth, linearly independent one-forms defined on $U$. If there exists a one-form $\pi \neq 0$ such that

$$
\begin{aligned}
d \alpha^{i} & \equiv-\alpha^{i+1} \wedge \pi \bmod \alpha^{1}, \ldots, \alpha^{i} \quad i=1, \ldots, s-1 \\
d \alpha^{s} & \neq 0 \bmod I
\end{aligned}
$$

then there exists a set of coordinates $x$ such that

$$
I=\left\{d x_{s}-x_{s-1} d x_{1}, \ldots, d x_{3}-x_{2} d x_{1}\right\} .
$$


Remark 1. A set of vector fields which are perpendicular to the ideal $I$ are given by

$$
g_{1}=\frac{\partial}{\partial x_{1}}+x_{2} \frac{\partial}{\partial x_{3}}+\cdots+x_{n-1} \frac{\partial}{\partial x_{n}} \quad g_{2}=\frac{\partial}{\partial x_{2}} .
$$

These are precisely the vector fields which appear in the two-input, single-chained form described in [13]. We say that a nonholonomic system with vector fields as in equation (6) is in chained form.

Let $\Sigma$ be a nonholonomic control system with smooth, independent, input vector fields $g_{1}$ and $g_{2}$. Define two filtrations

$$
\begin{aligned}
E_{0} & =\Delta & F_{0} & =\Delta \\
E_{1} & =E_{0}+\left[E_{0}, E_{0}\right] & F_{1} & =F_{0}+\left[F_{0}, F_{0}\right] \\
E_{2} & =E_{1}+\left[E_{1}, E_{1}\right] & F_{2} & =F_{1}+\left[F_{1}, F_{0}\right] \\
& \vdots & & \vdots \\
E_{i+1} & =E_{i}+\left[E_{i}, E_{i}\right] & F_{i+1} & =F_{i}+\left[F_{i}, F_{0}\right] .
\end{aligned}
$$

Under the assumption that each distribution is constant rank, the filtrations have finite length (possibly different).

The filtration $\left\{F_{i}\right\}$ is the the one which usually appears in the context of nonlinear controllability and feedback linearization. In particular, $F_{i}$ consists of all brackets up to order $i$. The distribution $E_{i}$ also contains all brackets of order $i$, but may contain additional Lie products of higher order. This is due to the recursive construction of $E_{i}$, as opposed to the iterative construction of $F_{i}$. The filtration $E_{i}$ is precisely the sequence of distributions which is perpendicular to the derived flag of $I=\Delta^{\perp}$.

Theorem 3. Given a 2-dimensional distribution $\Delta$ such that

$$
\operatorname{dim} E_{i}=\operatorname{dim} F_{i}=i+2 \quad i=0, \ldots, n-2
$$

there exists a local basis $\left\{\alpha^{1}, \ldots, \alpha^{s}\right\}$ and a one-form $\pi$ such that the Goursat congruences (5) are satisfied for the differential system $I=\Delta^{\perp}$.

A proof of this result, based on a suggestion by W. Sluis [14], is given in Appendix A. The fact that the dimension of the derived flag (or equivalently the filtration $\left\{E_{i}\right\}$ ) is not sufficient to guarantee equivalence to the Goursat normal form has been pointed out by Giaro, Kumpera, and Ruiz [4], who constructed a counterexample in 5 dimensions. They also note that if $\operatorname{dim} E_{i}=i+2$ then the Goursat conditions hold on an open dense set. For the applications considered here, the stronger result of Theorem 3 is needed.

2.3. Application to the control problem. The Goursat normal form can be used to give conditions under which a system is feedback transformable to a system in chained form. The following proposition follows immediately by application of Theorems 2 and 3. 
Proposition 4. There exists a feedback transformation which puts a nonholonomic system into chained form (6) if and only if

$$
\operatorname{dim} E_{i}=\operatorname{dim} F_{i}=i+2 \quad i=0, \ldots, n-2 .
$$

Thus, the class of two-input systems which can be converted into chained form (with a single chain) is completely characterized by an algebraic test. It is interesting to note that although chained systems are not generic, they occur frequently in applications. For example, all two-input, regular nonholonomic systems in 3 and 4 dimensions are locally feedback equivalent to a system in chained form. This follows from Darboux's theorem and Engel's theorem, respectively, and is a special case of the results presented above (see [2, pp. 50-51] for a proof of Engel's theorem). These special cases have also pointed out by Hermes [5]. Other systems, such as a car with 1 or 2 trailers also satisfy the conditions necessary to put them into chained form.$^{1}$

The results of Proposition 4 provide a set of sufficient conditions for the existence of a nilpotent basis for a two-dimensional distribution. The conditions are obviously not necessary since a distribution might admit a nilpotent basis even though the growth conditions in Proposition (4) are not satisfied. One simple example is

$$
\begin{aligned}
& g_{1}=\frac{\partial}{\partial x_{1}}+x_{2} \frac{\partial}{\partial x_{3}}+x_{3} \frac{\partial}{\partial x_{4}} \\
& g_{2}=\frac{\partial}{\partial x_{2}}+x_{3} \frac{\partial}{\partial x_{5}},
\end{aligned}
$$

which has a derived flag with dimensions $(3,2,0)$ and hence $\operatorname{dim} E_{i}=\operatorname{dim} F_{i} \neq i+2$. Even if we restrict the growth of the distribution so that $\operatorname{dim} F_{i}=i+2$ the conditions are only sufficient, as the following vector fields show:

$$
\begin{aligned}
& g_{1}=\frac{\partial}{\partial x_{1}}+x_{2} \frac{\partial}{\partial x_{3}}+x_{3} \frac{\partial}{\partial x_{4}} x_{4} \frac{\partial}{\partial x_{5}}+\left(x_{5}+x_{3}^{2}\right) \frac{\partial}{\partial x_{6}} \\
& g_{2}=\frac{\partial}{\partial x_{2}}
\end{aligned}
$$

For this distribution the filtration $\left\{F_{i}\right\}$ grows at the proper rate, but $E_{4}$ does not satisfy the dimension count.

One disadvantage of using Proposition 4 is the need to find an explicit change of coordinates which puts the system into chained form. Even in relatively low dimensional cases, the computations can become extremely complex.

Example 1 (Rolling Penny). Consider the kinematic model of a penny rolling on a plane, as shown in Figure 1. Let $x \in \mathbb{R}^{4}$ denote the configuration of the penny, with $\left(x_{1}, x_{2}\right)$ being the location of the penny on the plane, $x_{3}$ the angle that the penny

\footnotetext{
${ }^{1}$ The case of a car with $N$ trailers may also satisfy the conditions for conversion into chained form, but the calculations appear to require a high degree of organization which has thus far eluded the author.
} 


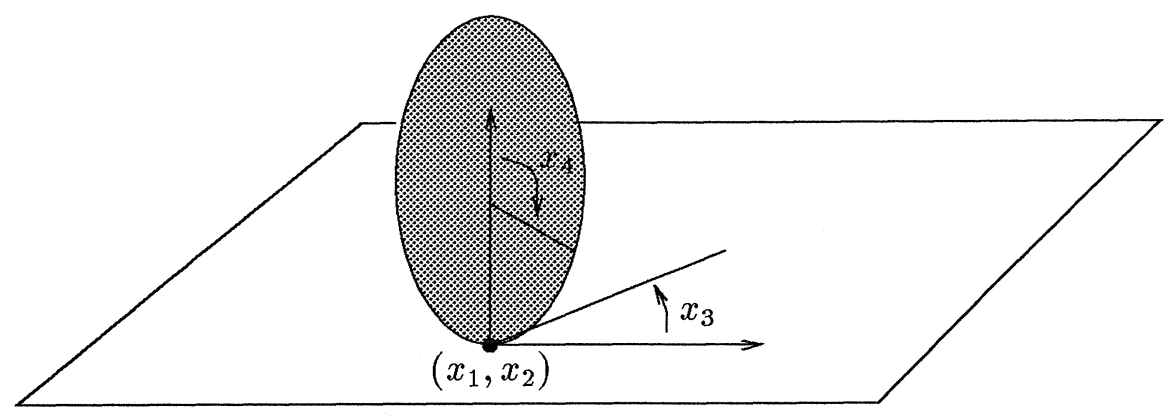

Figure 1. Penny rolling on a plane

makes with a fixed line on the plane, and $x_{4}$ the angle of a fixed line on the penny with respect vertical. We take the radius of the penny as 1 . The constraints for the penny are that it roll in the direction it is pointing, with no slipping:

$$
\begin{aligned}
& \alpha^{1}=\cos x_{3} d x_{1}+\sin x_{3} d x_{2}-d x_{4} \\
& \alpha^{2}=\sin x_{3} d x_{1}-\cos x_{3} d x_{2}
\end{aligned}
$$

Converting this to a control system gives

$$
\left[\begin{array}{c}
\dot{x}_{1} \\
\dot{x}_{2} \\
\dot{x}_{3} \\
\dot{x}_{4}
\end{array}\right]=\left[\begin{array}{c}
\cos x_{3} \\
\sin x_{3} \\
0 \\
1
\end{array}\right] u_{1}+\left[\begin{array}{l}
0 \\
0 \\
1 \\
0
\end{array}\right] u_{2}
$$

For this choice of vector fields, $u_{1}$ corresponds to the forward velocity of the penny and $u_{2}$ corresponds to the angular velocity of the penny about the plane's normal. A simple calculation shows that

$$
\operatorname{ad}_{g_{1}}^{4} g_{2}=-\operatorname{ad}_{g_{1}}^{2} g_{2}
$$

and hence these vector fields are not nilpotent. However, Proposition 4 can be used to find a change of input which makes the system nilpotent.

The first step is to compute the derived flag for the exterior differential system. The exterior derivatives of $\alpha^{1}$ and $\alpha^{2}$ are given by

$$
\begin{aligned}
& d \alpha^{1}=-\sin x_{3} d x_{3} \wedge d x_{1}+\cos x_{3} d x_{3} \wedge d x_{2} \\
& d \alpha^{2}=\cos x_{3} d x_{3} \wedge d x_{1}+\sin x_{3} d x_{3} \wedge d x_{2}
\end{aligned}
$$

It is easy to check that the following relationships hold

$$
\begin{gathered}
d \alpha^{1} \wedge \alpha^{1}=-d x_{1} \wedge d x_{2} \wedge d x_{3} \\
d \alpha^{1} \wedge \alpha^{1} \wedge \alpha^{2}=0 \\
d \alpha^{2} \wedge \alpha^{1} \wedge \alpha^{2}=-d x_{1} \wedge d x_{2} \wedge d x_{3} \wedge d x_{4} \neq 0
\end{gathered}
$$


and hence $\left\{\alpha^{1}, \alpha^{2}\right\}$ is a basis adapted to the derived flag:

$$
\begin{aligned}
& I^{(0)}=\left\{\alpha^{1}, \alpha^{2}\right\} \\
& I^{(1)}=\left\{\alpha^{1}\right\} \\
& I^{(2)}=\{\} .
\end{aligned}
$$

The dimension of the derived flag is $(2,1,0)$, implying that the system is completely controllable. Furthermore, it is easy to check that the conditions of Theorem 3 are satisfied and hence the system admits a nilpotent basis.

Next, we search for a one-form $\pi$ which satisfies the Goursat congruences. Since this system is low dimensional, the algebra needed to find $\pi$ is straightforward. Define $\alpha^{3}$ and $\alpha^{4}$ so as to complete the basis:

$$
\begin{aligned}
& \alpha^{3}=\cos x_{3} d x_{1}+\sin x_{3} d x_{2} \\
& \alpha^{4}=d x_{3} .
\end{aligned}
$$

$\pi$ must satisfy

$$
d \alpha^{1}=-\alpha^{2} \wedge \pi \bmod \alpha^{1}
$$

Setting $\pi=\lambda_{3} \alpha^{3}+\lambda_{4} \alpha^{4}$, equation (14) gives

$$
\begin{aligned}
& \lambda_{3}=0 \\
& \lambda_{4}=1
\end{aligned} \quad \Longrightarrow \quad \pi=d x_{3} .
$$

Following the proof of the Goursat theorem and solving a set of first-order partial differential equations (see [2]), the following change of coordinates is used to put the system into Goursat normal form:

$$
\begin{aligned}
& z_{1}=x_{3} \\
& z_{2}=-x_{1} \cos x_{3}-x_{2} \sin x_{3} \\
& z_{3}=-x_{1} \sin x_{3}+x_{2} \cos x_{3} \\
& z_{4}=x_{1} \cos x_{3}+x_{2} \sin x_{3}-x_{4}
\end{aligned}
$$

In this new set of coordinates, the one-forms become

$$
\begin{aligned}
& \alpha^{1}=d z_{4}-z_{3} d z_{1} \\
& \alpha^{2}=-\left(d z_{3}-z_{2} d z_{1}\right)
\end{aligned}
$$

and this is easily converted to a Goursat basis by negating $\alpha^{2}$. Let $\tilde{g}_{i}$ represent the chained vector fields in Goursat coordinates; in the original coordinates these vector fields have the form

$$
g_{i}(x)=\left(\frac{\partial \phi}{\partial x}\right)^{-1} \tilde{g}_{i}(\phi(x))
$$


where $\phi: x \mapsto z$ is the Goursat change of coordinates. Performing this calculation, the nilpotent control system has the form

$$
\left[\begin{array}{c}
\dot{x}_{1} \\
\dot{x}_{2} \\
\dot{x}_{3} \\
\dot{x}_{4}
\end{array}\right]=\left[\begin{array}{c}
-\cos x_{3}\left(x_{2} \cos x_{3}-x_{1} \sin x_{3}\right) \\
-\sin x_{3}\left(x_{2} \cos x_{3}-x_{1} \sin x_{3}\right) \\
1 \\
-\left(x_{2} \cos x_{3}-x_{1} \sin x_{3}\right)
\end{array}\right] v_{1}+\left[\begin{array}{c}
-\cos x_{3} \\
-\sin x_{3} \\
0 \\
-1
\end{array}\right] v_{2} .
$$

Here $v_{2}$ corresponds to the negative of the angular velocity of the penny about the plane's normal and $v_{1}$ is a linear combination of $v_{2}$ and the forward velocity of the penny.

2.4. Systems with more than two inputs. Partial extensions of the results presented above to systems with more than two inputs are available using the recent work of Gardner and Shadwick [3]. Gardner and Shadwick have proposed an algorithm for MIMO feedback linearization which uses an extension of the Goursat theorem presented above.

Theorem 5 (Extended Goursat Normal Form). Let $U$ be an open subset of $\mathbb{R}^{n}$ and $I=\left\{\alpha_{j}^{1}, \ldots, \alpha_{j}^{s_{j}}: j=1, \ldots, m-1\right\}$ be a collection of smooth, linearly independent one-forms defined on $U$. Let $I^{(j)}$ denote the $j^{\text {th }}$ derived system of $I$. If there exists a single one-form $\pi \neq 0$ such that for all $j$

$$
\begin{aligned}
& d \alpha_{j}^{i} \equiv-\alpha_{j}^{i+1} \wedge \pi \bmod I^{\left(s_{j}-i\right)} \quad i=1, \ldots, s_{j}-1 \\
& d \alpha_{j}^{s_{j}} \neq 0 \quad \bmod I
\end{aligned}
$$

then there exists a set of coordinates $x$ such that

$$
I=\left\{d x_{s}^{j}-x_{s-1}^{j} d x_{1}, \ldots, d x_{3}^{j}-x_{2}^{j} d x_{1}\right\} .
$$

Remark 2. A set of vector fields which is perpendicular to the ideal $I$ is given by

$$
g_{1}=\frac{\partial}{\partial x_{1}}+x_{2}^{j} \frac{\partial}{\partial x_{3}^{j}}+\cdots+x_{n-1}^{j} \frac{\partial}{\partial x_{n}^{j}} \quad g_{j+1}=\frac{\partial}{\partial x_{2}^{j}} .
$$

These vector fields are a special case of the multi-input chained form vector fields described in [13].

The exterior differential system $I_{j}=\left\{\alpha_{j}^{1}, \ldots, \alpha_{j}^{s_{j}}\right\}$ is called the $j^{\text {th }}$ tower of the complete system. Theorem 5 is considerably more restrictive than the original Goursat theorem since it requires a very limited coupling between the different towers. This is enforced by the independence of the mod operations in Theorem 5 and the use of a single one-form $\pi$ which connects the towers. In the case MIMO feedback linearization, $\pi$ becomes $d t$, the differential of time.

In the two-input case, Theorem 3 was used to show that the Goursat conditions could always be satisfied if a certain dimension count was satisfied. This is not true when more than two inputs are present, since the growth of control Lie algebra can be much more complex. 


\section{Application: trajectory generation}

The results of the previous section can be used to generate a feedback transformation which renders a nonholonomic control system nilpotent. Recent work by Lafferriere and Sussmann [10] and Jacob [7, 8] has given insights into how to generate trajectories for nilpotent control systems. We review those results here and combine them with some of the steering methods presented in [13] to obtain a technique for trajectory generation for chained systems. The methods presented here do not rely on finding the coordinate transformation which puts the system explicitly into chained form, but only on the input transformation which renders the system nilpotent.

Following the results of the previous section, we consider control systems of the form

$$
\dot{x}=g_{1}(x) u_{1}+\cdots+g_{m}(x) u_{m}
$$

where the vector fields

$$
\begin{aligned}
& g_{1}, \\
& g_{2}, \operatorname{ad}_{g_{1}} g_{2}, \operatorname{ad}_{g_{1}}^{2} g_{2}, \ldots, \operatorname{ad}_{g_{1}}^{n_{2}} g_{2} \\
& \vdots \\
& g_{m}, \operatorname{ad}_{g_{1}} g_{m}, \operatorname{ad}_{g_{1}}^{2} g_{m}, \ldots, \operatorname{ad}_{g_{1}}^{n_{m}} g_{m}
\end{aligned}
$$

span $T_{x} M, n=1+n_{2}+\cdots+n_{m}$, at each point $x \in U$ and all other Lie products between the vector fields are identically zero. These are precisely the class of systems which are generated by the chained form theorems of the previous section and hence constructive, sufficient conditions for finding a change of basis (precompensator) which renders the system nilpotent are available.

To steer nilpotent, nonholonomic control systems with chained structure, we use exponential coordinates. Let $\phi_{h}^{f}$ represent the flow along a vector field $f$ for $h$ units of time. If $f$ is smooth and everywhere nonzero, $\phi_{h}^{f}$ exists for $h$ sufficiently small and $\phi_{h}^{f}: \mathbb{R}^{n} \rightarrow \mathbb{R}^{n}$ is a diffeomorphism of the state space onto itself. The flow satisfies the differential equation

$$
\frac{\partial}{\partial h} \phi_{h}^{f}=f \circ \phi_{n}^{f}
$$

Now let $\left\{B_{1}, \ldots, B_{r}\right\}$ be a P. Hall basis of order $k$ generated by the symbols $g_{i}$. We let $b_{i}$ denote a basis element $B_{i}$ which has been evaluated on the vector fields $g_{1}, \ldots, g_{m}$. Concatenating the flows of the $B_{i}$ we obtain a map $\Phi: h \mapsto x$,

$$
\Phi(h)=\phi^{b_{1} h_{1}} \cdots \phi^{b_{r} h_{r}}\left(x^{0}\right) .
$$

This map is surjective in a neighborhood of $h=0$ and is a local diffeomorphism in the case that $r=n$. By an abuse of terminology, we call $h$ the exponential coordinates adapted to the basis $\left\{B_{i}\right\}$ (even though $\Phi$ is not necessarily injective).

Our goal is to find an $h^{1}$ such that $x^{1}=\Phi\left(h^{1}\right)$ and then solve the problem in $h$ coordinates. This entails finding a $u$ such that the original system is steered to 
the desired $h$ coordinates. The power of this approach is that we never have to explicitly solve for the mapping $\Phi$. It suffices to consider the differential equation which $h$ must satisfy and solve the problem in that context. Computationally, this can be done in such a way as to require nothing more than simple integration and the solution of algebraic (typically polynomial) equations. The foundations of this technique can be found in the work of Sussmann $[15,10]$ and have been used in much the same form as given here in the recent work of Jacob [8]. We present only the case where the Lie algebra generated by $g_{1}$ and $g_{2}$ has the chained structure derived in the previous section, although the general case follows in essentially the same way.

To compute the value $h(1)$ which corresponds to the desired final state of the original system, we make use of the "extended system" defined by Lafferriere and Sussmann:

$$
\dot{x}=b_{1}(x) v_{1}+b_{2}(x) v_{2}+\cdots+b_{r}(x) v_{r} .
$$

By assumption, the vector fields $b_{1}, \ldots, b_{r}$ are full rank. Given any path $x(t)$, we can solve for some $v(t)$ which satisfies equation (19). This corresponds to choosing how to project the velocity of the system onto the P. Hall basis elements; if $r=n$ this choice is unique, otherwise not. We discuss some possibilities about how the extra freedom in the $r>n$ case might be exploited in the discussion section.

We can find the exponential coordinates corresponding to this motion by differentiating equation (18). This yields a set of differential equations for $h_{i}$, as follows:

$$
\begin{aligned}
\dot{h}_{1} & =v_{1} \\
\dot{h}_{2} & =v_{2} \\
\dot{h}_{3} & =h_{2} v_{1}+v_{3} \\
\dot{h}_{4} & =\frac{1}{2} h_{2}^{2} v_{1}+h_{2} v_{3}+v_{4} \\
& \vdots \\
\dot{h}_{n} & =\frac{1}{(n-2) !} h_{2}^{n-2} v_{1}+\cdots+h_{2} v_{n-1}+v_{n}
\end{aligned}
$$

A complete derivation of these equations can be found in [15]. Since $v(t)$ is known, this equation can be integrated to find $h(1)$.

The task is now to find a feasible path which steers the system to the same point in exponential coordinates. Since every feasible path corresponds to one in which $v_{j}=0, j>m$, the problem reduces to finding $v_{1}, \ldots, v_{m}$ which steers $(20)$ from $h(0)=0$ to $h(1)=h^{1}$. The simple form of equation (20) allows us to choose $v(t)$ as a parameterized function (e.g. a Fourier or Taylor series in $t$ ), integrate the differential equation to obtain a parameterized final state, and equate the final state to the desired state. For Fourier and Taylor series parameterizations of the inputs, the final solution can be reduced to finding the roots of a polynomial. We illustrate this in an example below. 
The system (20) can be shown to be diffeomorphic to a system in chained form [16]. Hence, the choice of $v(t)$ can be transformed into the problem of solving the trajectory generation problem for a system in chained form. Specific algorithms for steering systems in chained form are presented in [13].

The algorithm outlined here can be extended to the case of an arbitrary nilpotent Lie algebra instead of the special chained form used here. In this case one again proceeds by using exponential coordinates and solving a polynomial control problem relative to that basis. The general case is discussed in $[10,8]$.

Example 2. Consider again the rolling penny from the previous example. Let $x^{0}=$ $(0,0,0,0)$ be the initial configuration and let $x^{1}=(1,2,-\pi / 2, \pi / 4)$ be the desired final configuration. Motion between $x^{0}$ and $x^{1}$ consists of a net translation in the $x$ and $y$ directions, combined with a $90^{\circ}$ rotation of the penny relative to the a fixed line on the plane and a $45^{\circ}$ degree rotation of a fixed line on the penny with respect to vertical. We assume that system is nilpotent, as in equation (15)

We begin by constructing the exponential coordinates corresponding to $x^{1}$. Since the P. Hall basis evaluated on the system vector fields has exactly 4 non-zero elements, the extended system is uniquely defined as

$$
\dot{x}=g_{1} v_{1}+g_{2} v_{2}+\left(\operatorname{ad}_{g_{1}} g_{2}\right) v_{3}+\left(\operatorname{ad}_{g_{1}}^{2} g_{2}\right) v_{4} .
$$

Choosing $x(t)=x^{0}+t\left(x^{1}-x^{0}\right)$ we can solve for $v(t)$ and substitute integrate equation (20) to determine the exponential coordinates corresponding to $x^{1}$. Performing this calculation yields $h^{1} \approx(-1.57,1,-3.57,2.59)$.

We now construct an input which steers the system in exponential coordinates from $h^{0}$ to $h^{1}$. Using the ideas developed in [13], we choose

$$
\begin{aligned}
& v_{1}=a_{0} \sin \pi t+a_{1} \sin 2 \pi t \\
& v_{2}=b_{0} \sin \pi t+b_{1} \cos 2 \pi t
\end{aligned}
$$

which can be integrated symbolically (from the origin) to obtain:

$$
\begin{aligned}
& h_{1}(1)=\frac{2}{\pi} a_{0} \\
& h_{2}(1)=\frac{2}{\pi} b_{0} \\
& h_{3}(1)=\frac{2}{\pi^{2}} a_{0} b_{0}+\frac{4}{3 \pi^{2}} a_{1} b_{0}+\frac{8}{15 \pi^{2}} a_{2} b_{0}-\frac{1}{4 \pi} a_{1} b_{1} \\
& h_{4}(1)=\frac{4}{3 \pi^{3}} a_{0}^{2} b_{0}+\frac{4}{3 \pi^{3}} a_{0} a_{1} b_{0}+\frac{8}{15 \pi^{2}} a_{1}^{2} b_{0}-\frac{1}{8 \pi^{2}} a_{0}^{2} b_{1}-\frac{1}{4 \pi^{2}} a_{0} a_{1} b_{1}-\frac{1}{8 \pi^{2}} a_{1}^{2} b_{1} .
\end{aligned}
$$

This is a polynomial equation in the input parameters. A solution of the equation $h(1)=h^{1}$ (obtained using Mathematica) is given by

$$
\begin{aligned}
& v_{1}=-2.467 \sin \pi t-2.273 \sin 2 \pi t \\
& v_{2}=1.571 \sin \pi t-12.73 \cos 2 \pi t .
\end{aligned}
$$

The motion generated by this set of inputs is shown in Figure 2. 

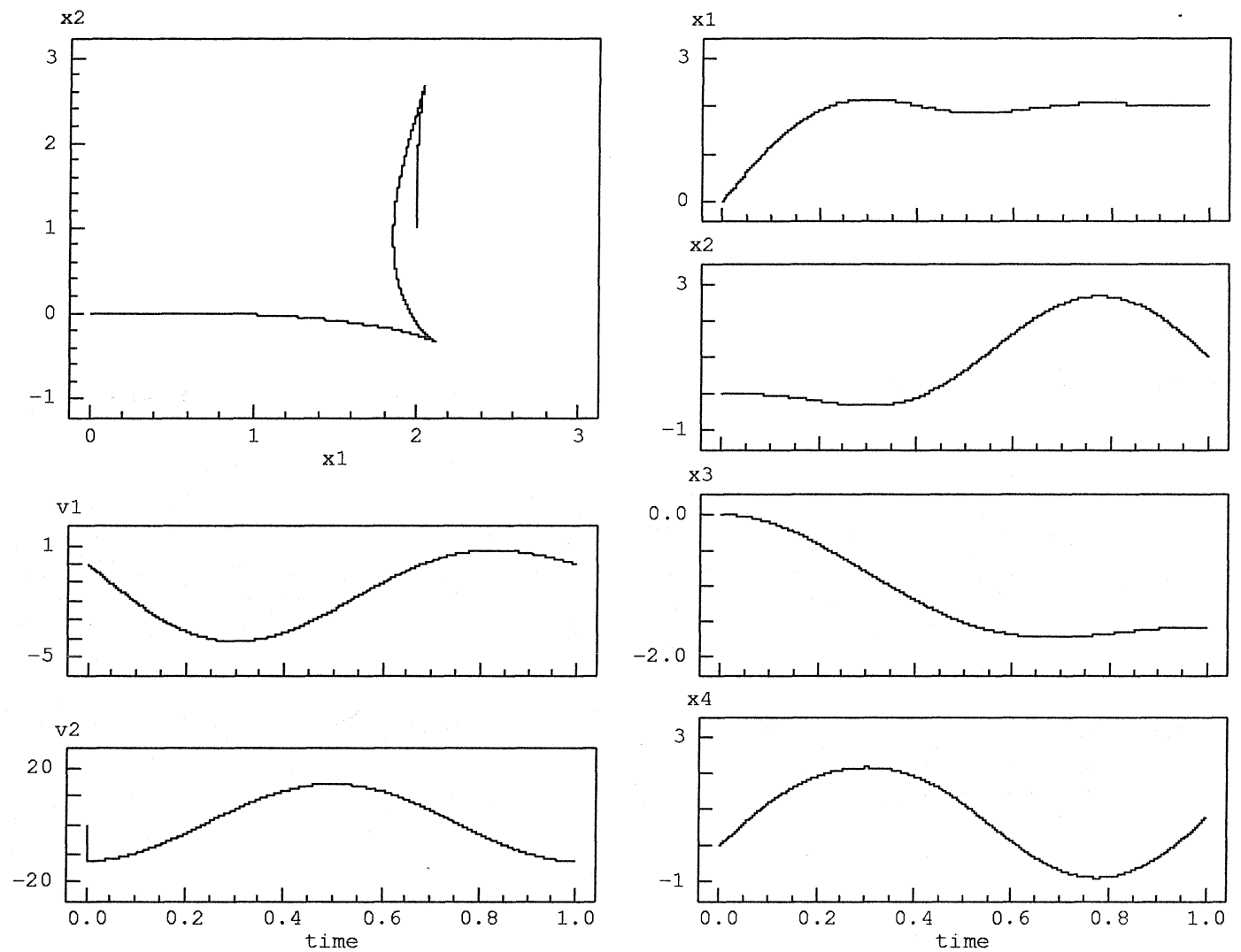

FIGURE 2. Motion of a rolling penny from $x^{0}=(0,0,0,0)$ to $x^{1}=$ $(2,1, \pi / 2, \pi / 4)$. The upper left figure shows the trajectory of the penny on the plane; the remaining plots show the states and inputs as a function of time.

\section{Conclusions}

In this paper we have presented a set of necessary and sufficient conditions for converting a two-input, nonholonomic control system into a special canonical form. This result also gives constructive sufficient conditions for finding a nilpotent basis for a nonholonomic distribution. The application of these results to steering a control system with a nilpotent basis was also given.

Further research is needed to achieve an understanding of the conditions under which a nonholonomic system with more than two inputs can be made nilpotent by use of a nonlinear precompensator. The extended Goursat normal form theorem used by Gardner and Shadwick gives one criterion but there are many other possibilities. As an example, all systems which are in chained form (in its full generality, as described in [13]) are nilpotent, but only a very special subset satisfy the extended Goursat normal form conditions. They correspond to systems in which controllability is "generated" by a single vector field (which plays the dual role of the one-form $\pi$ ). 
As noted by Jacob [8], path planning techniques based on exponential coordinates generated by a nilpotent basis do not rely on the system having no drift vector field. The same techniques can be applied to systems of the form

$$
\dot{x}=f(x)+g_{1}(x) u_{1}+\cdots g_{m}(x) u_{m}
$$

if the vector fields are nilpotent. However, the problem of finding a nilpotent basis for a nonlinear control system which is only affine in the inputs is considerably more difficult, since the drift vector field $f$ cannot be scaled. It is interesting to note that the Brunovsky canonical form used in feedback linearization is nilpotent, and hence the MIMO linearization conditions are sufficient conditions for finding a nilpotent basis for a nonlinear system which is affine in the inputs.

Acknowledgements. The author would like to thank R. Gardner and W. Sluis of the Fields Institute for Mathematical Sciences, Ontario, Canada for many fruitful conversations on the use of exterior differential systems for studying chained systems and for pointing out the connections between Goursat normal form and chained systems. In addition, Sameer Jalnapurkar and Michiel van Nieuwstadt provided invaluable assistance in debugging the proof for Theorem 3.

\section{REFERENCES}

1. A. M Bloch and N. H. McClamroch. Control of mechanical systems with classical nonholonomic constraints. In IEEE Control and Decision Conference, pages 201-205, 1989.

2. R. L Bryant, S. S. Chern, R. B. Gardner, H. L. Goldschmidt, and P. A. Griffiths. Exterior Differential Systems. Springer-Verlag, 1991.

3. R. B. Gardner and W. F. Shadwick. The GS algorithm for exact linearization to Brunovsky normal form. IEEE Transactions on Automatic Control, 37(2):224-230, 1992.

4. A. Giaro, A. Kumpera, and C. Ruiz. Sur la lecture correcte d'un résultat d'Éli Cartan. Comptes Rendus des Séances de l'Académie des Sciences, 287, Série A:241-244, 1978.

5. H. Hermes. Distributions and the Lie algebras their bases can generate. Proceedings of the American Mathematical Society, 106(2):555-565, 1989.

6. H. Hermes, A. Lundell, and D. Sullivan. Nilpotent bases for distributions and control systems. Journal of Differential Equations, 55:385-400, 1984.

7. G. Jacob. Lyndon discretization and exact motion planning. In European Control Conference, 1991.

8. G. Jacob. Motion planning by piecewise constant or polynomial inputs. In IFAC Symposium on Nonlinear Control Systems Design (NOLCOS), pages 628-633, 1992.

9. G. Lafferriere and H. J. Sussmann. Motion planning for controllable systems without drift: a preliminary report. Technical Report SYCON-90-04, Rutgers Center for Systems and Control, 1990.

10. G. Lafferriere and H. J. Sussmann. Motion planning for controllable systems without drift. In IEEE International Conference on Robotics and Automation, pages 1148-1153, 1991. 
11. J-P. Laumond, P. Jacobs, M. Taix, and R. M. Murray. A motion planner for nonholonomic mobile robots. IEEE Transactions on Robotics and Automation, 1992. (to appear).

12. R. M. Murray and S. S. Sastry. Steering nonholonomic systems in chained form. In IEEE Control and Decision Conference, pages 1121-1126, 1991.

13. R. M. Murray and S. S. Sastry. Nonholonomic motion planning: Steering using sinusoids. IEEE Transactions on Automatic Control, 1992. (to appear).

14. W. Sluis. Personal Communication, June 1992.

15. H. J. Sussmann. A product expansion for the Chen series. In C. Byrnes and A. Lindquist, editors, Theory and Applications of Nonlinear Control Systems, pages 323-335. North-Holland, 1986.

16. A. Teel, R. M. Murray, and G. Walsh. From steering to stabilization with sinusoids. In IEEE Control and Decision Conference, 1992. (to appear). 


\section{Appendix A. Proof of Theorem 3}

Theorem 3. Given a 2-dimensional distribution $\Delta$ such that

$$
\operatorname{dim} E_{i}=\operatorname{dim} F_{i}=i+2 \quad i=0, \ldots, n-2
$$

there exists a basis $\left\{\alpha^{1}, \ldots, \alpha^{s}\right\}$ and a one-form $\pi$ such that the Goursat congruences are satisfied for the differential system $I=E_{0}^{\perp}$.

The proof relies on the following lemma.

Lemma 6. Under the assumptions of Theorem 3 there exists a basis $\left\{\alpha^{1}, \ldots, \alpha^{s}\right\}$ and a set of one-forms $\lambda^{i}$ such that $\lambda^{i} \bmod I \neq 0$ and

$$
\begin{aligned}
d \alpha^{1} & \equiv \lambda^{1} \wedge \alpha^{2} & & \bmod \alpha^{1} \\
d \alpha^{2} & \equiv \lambda^{2} \wedge \alpha^{3} & & \bmod \alpha^{1}, \alpha^{2} \\
& \vdots & & \\
d \alpha^{s-1} & \equiv \lambda^{s-1} \wedge \alpha^{s} & & \bmod \alpha^{1}, \ldots, \alpha^{s-1} \\
d \alpha^{s} & \neq 0 & & \bmod I
\end{aligned}
$$

Proof. Choose a basis for $I$ which is adapted to the derived flag:

$$
I^{(i)}=\left\{\alpha^{1}, \ldots, \alpha^{s-i}\right\} .
$$

Since $\left\{\alpha^{i}\right\}$ is adapted to the derived flag, we have immediately that the congruences (24) are satisfied and $d \alpha^{i} \neq 0 \bmod \alpha^{1}, \ldots, \alpha^{i}$. For later use, we also note that any transformation of the form

$$
\bar{\alpha}^{i}=a^{i} \alpha^{i}+\sum_{j<i} b_{j}^{i} \alpha^{j} \quad a^{i}>0
$$

also satisfies the congruences (24) with $\bar{\lambda}^{i}=\left(a^{i} / a^{i+1}\right) \lambda^{i}$. It follows from the definition of $\left\{E_{i}\right\}$ that $\alpha^{s-i}\left(E_{j}\right)=0$ for $j \leq i$ and that for any $X, Y \in E_{i}$

$$
\begin{aligned}
d \alpha^{s-i}(X, Y) & =X \alpha^{s-i}(Y)-Y \alpha^{s-i}(X)-\alpha^{s-i}([X, Y]) \\
& =\alpha^{s-i}([X, Y]) .
\end{aligned}
$$

Since $\alpha^{s-i} \notin I^{(i+1)}$ then there exists some $X, Y \in E_{i}$ such that $[X, Y] \notin E_{i}$ and $d \alpha^{s-i}(X, Y) \neq 0$.

We show $\lambda^{s-i} \bmod I \neq 0$ by contradiction. First note that $F_{i} \subset E_{i}$ and since they have the same dimension we must have $F_{i}=E_{i}$. This in turn implies that

$$
E_{i+1}=E_{i}+\left[E_{i}, E_{0}\right]
$$

and hence every $Z \in E_{i+1}$ such that $Z \notin E_{i}$ has the form $Z=[X, Y]$ for some $X \in E_{i}, Y \in E_{0}$. Thus there exists some such $X, Y$ with

$$
d \alpha^{s-i}(X, Y)=\alpha^{s-i}(Z) \neq 0 .
$$


Now if $\lambda^{s-i} \bmod I=0$ then $\lambda^{s-i}(Y)=0$ and

$$
\begin{aligned}
d \alpha^{s-i}(X, Y) & =\left(\lambda^{s-i} \wedge \alpha^{s-i+1}\right)(X, Y) \\
& =\lambda^{s-i}(X) \alpha^{s-i+1}(Y)-\lambda^{s-i}(Y) \alpha^{s-i+1}(X) \\
& =0
\end{aligned}
$$

which is a contradiction. Hence $\lambda^{s-i} \bmod I \neq 0$.

Proof of theorem. Choose a basis for $I$ as in the proof of Lemma 6 . If we define $\pi=\lambda^{s-1}$ then equation (24) has the desired form down to $i=s-1$ and $\pi$ is determined $\bmod \alpha^{1}, \ldots, \alpha^{s}$. We now proceed by induction. Assume

$$
d \alpha^{i}=\pi \wedge \alpha^{i+1} \bmod \alpha^{1}, \ldots, \alpha^{i}
$$

and that $\pi$ is determined $\bmod \alpha^{1}, \ldots, \alpha^{i+1}$. Dropping the superscript on $\lambda^{i-1}$ we have

$$
d \alpha^{i-1} \equiv \lambda \wedge \alpha^{i} \quad \bmod \alpha^{1}, \ldots, \alpha^{i-1}
$$

and taking the exterior derivative and wedging with $\alpha^{i}$ gives

$$
\left(d^{2} \alpha^{i-1}\right) \wedge \alpha^{i}=0= \pm \lambda \wedge \pi \wedge \alpha^{i} \wedge \alpha^{i+1} \bmod \alpha^{1}, \ldots, \alpha^{i-1}
$$

and hence

$$
\lambda=a \pi+b \alpha^{i}+c \alpha^{i+1} \bmod \alpha^{1}, \ldots, \alpha^{i-1} .
$$

From the lemma we have that $\lambda \bmod I \neq 0$ and therefore $a \neq 0$. Hence we can define a new one-form $\bar{\pi}$ as

$$
\bar{\pi}=a \pi+b \alpha^{i}+c \alpha^{i+1} .
$$

By scaling $\alpha^{i}, \ldots, \alpha^{s}$, the congruences down to $i$ are preserved with $\bar{\pi}$ replacing $\pi$. Furthermore, we obtain

$$
d \alpha^{i-1} \equiv \bar{\pi} \wedge \alpha^{i} \quad \bmod \alpha^{1}, \ldots, \alpha^{i-1}
$$

and $\bar{\pi}$ is determined mod $\alpha^{1}, \ldots, \alpha^{i}$. This completes the induction.

To explore the need for the assumption on the dimension of $F_{i}$, we further examine the relationship

$$
\bar{\pi}=a \pi+b \alpha^{i}+c \alpha^{i+1} .
$$

Without loss of generality we can take $b=0$ since it does not affect the congruences at this level. If we do not require $\lambda \bmod I \neq 0$, then we cannot define $\bar{\pi}$ as before. There are two possibilities: $a \equiv 0$ or $a=0$ at a point. It turns out that $a \equiv 0$ leads to a contradiction. To show this, we assume that $c=1$ by scaling the $\alpha^{i}$ 's appropriately, and hence we have

$$
d \alpha^{i-1} \equiv \alpha^{i+1} \wedge \alpha^{i} \bmod \alpha^{1}, \ldots, \alpha^{i-1} .
$$

We apply the standard trick: take the exterior derivative and analyze the resulting relationships. Taking the exterior derivative

$$
0=d^{2} \alpha^{i-1} \equiv d \alpha^{i+1} \wedge \alpha^{i}-\alpha^{i+1} \wedge d \alpha^{i}+\rho \wedge \alpha^{i+1} \wedge \alpha^{i} \quad \bmod \alpha^{1}, \ldots, \alpha^{i-1},
$$


where the additional term accounts for the possibility of adding a $d \alpha^{i-1}$ term due to the congruence. Now take the wedge product with $\alpha^{i+1}$ to obtain

$$
d \alpha^{i+1} \wedge \alpha^{i} \wedge \alpha^{i+1} \equiv 0 \quad \bmod \alpha^{1}, \ldots, \alpha^{i-1} .
$$

Shifting the wedge products into the mod, we have

$$
d \alpha^{i+1} \equiv 0 \quad \bmod \alpha^{1}, \ldots, \alpha^{i}, \alpha^{i+1},
$$

but this contradicts the fact that $d \alpha^{i+1} \neq 0 \bmod \alpha^{1}, \ldots, \alpha^{i+1}$ and hence $a \not \equiv 0$.

Thus the only possibility is that $a=0$ at a point (or on a submanifold), but not in a neighborhood. The extra assumptions on the dimension of the iterative filtration $\left\{F_{i}\right\}$ insure that this does not happen. This is illustrated in the following example, which is similar to that constructed by Giaro, Kumpera, and Ruiz [4].

Example 3. Consider the following distribution:

$$
\begin{aligned}
g_{1} & =\frac{\partial}{\partial x_{1}}+x_{2} \frac{\partial}{\partial x_{3}}+x_{3} \frac{\partial}{\partial x_{4}}+x_{2} x_{4} \frac{\partial}{\partial x_{5}} \\
g_{2} & =\frac{\partial}{\partial x_{2}}
\end{aligned}
$$

The filtrations corresponding to this distribution are defined in terms of the brackets

$$
\begin{gathered}
\operatorname{ad}_{g_{1}} g_{2}=-\frac{\partial}{\partial x_{3}}-x_{4} \frac{\partial}{\partial x_{5}} \quad \operatorname{ad}_{g_{1}}^{2} g_{2}=\frac{\partial}{\partial x_{4}}-x_{3} \frac{\partial}{\partial x_{5}} \quad \operatorname{ad}_{g_{2}}^{2} g_{1}=0 \\
\operatorname{ad}_{g_{1}}^{3} g_{2}=-2 x_{2} \frac{\partial}{\partial x_{5}} \quad\left[\operatorname{ad}_{g_{1}} g_{2}, \operatorname{ad}_{g_{1}}^{2} g_{2}\right]=2 \frac{\partial}{\partial x_{5}} .
\end{gathered}
$$

The filtrations $\left\{E_{i}\right\}$ and $\left\{F_{i}\right\}$ match for $i=0,1,2$. At the next level we have

$$
\begin{aligned}
& F_{3}=F_{2}+\left\{\operatorname{ad}_{g_{1}}^{3} g_{2}\right\} \\
& E_{3}=F_{2}+\left\{\operatorname{ad}_{g_{1}}^{3} g_{2},\left[\operatorname{ad}_{g_{1}} g_{2}, \operatorname{ad}_{g_{1}}^{2} g_{2}\right]\right\}
\end{aligned}
$$

The filtration $\left\{E_{i}\right\}$, which is perpendicular to the derived flag, has constant dimension $(2,3,4,5)$ but the distribution $F_{3}$ looses rank on the submanifold $x_{2}=0$. Hence there does not exist a basis and a one-form $\pi$ such that the Goursat congruences are satisfied. This shows the nature of the obstruction that can occur when the dimension count is correct only for the Goursat filtration $\left\{E_{i}\right\}$. 